\title{
VTI Media qP-wave Equation Using ONAD Method
}

\author{
Xiaochun $\mathrm{Ge}^{1, \mathrm{a}}$, Rong Huang ${ }^{1, \mathrm{~b}}$ and Guojie Song ${ }^{1, \mathrm{c}}$ \\ ${ }^{1}$ Shool of Sciences of Southwest Petroleum University, China \\ alion881011@126.com, bhr01101020@163.com, ccylsgj@126.com
}

Keywords: qP wave equation, forward simulation, ONAD method.

Abstract. The qP-wave equation is one of extremely important question in seismic exploration, seismology, and so on. However, its poor stability, the traditional finite difference scheme for qP-wave equation modeling suffer instability easily and numerical dispersion seriously. This paper propose a novel difference scheme for qP-wave simulation, named ONAD method. The numerical results demonstrate that, relative to LWC, the ONAD method for $\mathrm{qP}$-wave has obvious advantages. It's suitable for qP-wave modeling.

\section{Introduction}

Many studies proved that seismic velocity anisotropy is widely existed in underground media. In earlier studies, researchers suppose that the earth was isotropic which brought some uncertainty mistake for seismology interpretation. In order to represent anisotropic and simplify computational cost, Alkhalifah proposed the well-known acoustic approximation[1]. The so-called acoustic approximation set the shear wave velocity which is along the direction of symmetric axis to zero, then a approximate $\mathrm{qP}$-wave dispersion relation in anisotropic medium is obtained [2]. However, this processing led a four order partial differential equation in temporal-spatial domain which is very complicated and inconvenient. Aiming at a simple, stable governing equation, Zhou et al.[3]、Duveneck et al.[4] and Du et al.[5] derived coupled qP-wave equation by constructing auxiliary functions.

The qP-wave propagation simulation in the earth is the forward modeling of $\mathrm{qP}$-wave equations Many numerical algorithms can be used to solve the qP-wave equations, just like the finite-difference method, the finite-element method, the pseudo-spectrum method, and so on. Compared to the pseudo-spectrum method and the finite-element method, the finite difference method have become the most popular and widely used numerical methods in computational geophysics and petroleum exploration for theirs smaller memory cost ,fast computing speed, and high efficiency. However, when the conventional finite difference methods are used to simulation wave propagation with coarse grids, strong numerical dispersion will be aroused. The numerical dispersion is an important annoying shortcomings of numerical method.

Yang et. al. first proposed the so called NAD method and applied this method to the acoustic and elastic- wave field simulation [6]. NAD method reconstruct the wave-field by combining the displacement and its gradient to approximating high-order spatial partial derivative. From the mathematical point of view, the gradient represented the tendency of waveform change. Therefore increased gradient information can effectively enhance the ability of numerical schemes to suppress the numerical dispersion. ONADM is one of the perfect of NAD-type algorithms[7], but it was not to used for $\mathrm{qP}$-wave simulation. In this paper, we will expand the ONADM algorithm to qP wave forward modeling.

\section{qP wave equations in VTI medium and ONAD method}

According to phase velocity dispersion relation[8],

$$
\frac{V^{2}(\theta)}{V_{P 0}^{2}}=1+\varepsilon \sin ^{2} \theta-\frac{f}{2}+\frac{f}{2} \sqrt{\left(1+\frac{2 \varepsilon \sin ^{2} \theta}{f}\right)^{2}-\frac{8(\varepsilon-\delta) \sin ^{2} \theta \cos ^{2} \theta}{f}}
$$

Where, $f=1-\frac{V_{S 0}^{2}}{V_{P 0}^{2}}, V(\theta)$ is the $\mathrm{P}$-wave phase velocity, $V_{P 0}$ and $V_{S 0}$ are $\mathrm{qP}$-wave and qS-wave 
velocity along the symmetry axis, respectively. $\varepsilon$ and $\delta$ is Thomsen dimensionless anisotropy parameters[9].

According to the acoustic approximation $v_{S 0}=0$, the phase velocity relationship (1) can be simplified:

$$
V^{2}(\theta)=\frac{V_{P 0}^{2}}{2}\left(1+2 \varepsilon \sin ^{2} \theta\right)+\frac{V_{P 0}^{2}}{2} \sqrt{\left(1+2 \varepsilon \sin ^{2} \theta\right)^{2}-8(\varepsilon-\delta) \sin ^{2} \theta \cos ^{2} \theta}
$$

Based on equation(2), we follow the Duveneck's work and consider the two order coupled qP wave equations (3) in the rest of this paper.

$$
\frac{1}{V^{2}} \frac{\partial^{2}}{\partial t^{2}}\left(\begin{array}{l}
p \\
r
\end{array}\right)=\left(\begin{array}{cc}
1+2 \varepsilon & \sqrt{1+2 \delta} \\
\sqrt{1+2 \delta} & 1
\end{array}\right)\left(\begin{array}{cc}
\frac{\partial^{2}}{\partial x^{2}}+\frac{\partial^{2}}{\partial y^{2}} & 0 \\
0 & \frac{\partial^{2}}{\partial z^{2}}
\end{array}\right)\left(\begin{array}{l}
p \\
r
\end{array}\right)
$$

$p$ and $r$ are defined as the horizontal and vertical stress components, respectively.

According to the truncated Taylor expansion, we can get

$$
\left\{\begin{array}{l}
p_{i, j}^{n+1}=2 p_{i, j}^{n}-p_{i, j}^{n-1}+\Delta t^{2} c^{2}\left(\frac{\partial^{2} p}{\partial t^{2}}\right)+\frac{\Delta t^{4}}{12}\left(\frac{\partial^{4} p}{\partial t^{4}}\right) \\
r_{i, j}^{n+1}=2 r_{i, j}^{n}-r_{i, j}^{n-1}+\Delta t^{2} c^{2}\left(\frac{\partial^{2} r}{\partial t^{2}}\right)+\frac{\Delta t^{4}}{12}\left(\frac{\partial^{4} r}{\partial t^{4}}\right)
\end{array}\right.
$$

Similarly, following the idea of Dablain[11], we can convert temporal derivatives of formula (4) into spatial derivatives as

$$
\left\{\begin{aligned}
P_{i, j}^{n+1}= & 2 P_{i, j}^{n}-P_{i, j}^{n-1}+\Delta t^{2} V^{2}\left\{A \frac{\partial^{2} p}{\partial x^{2}}+B \frac{\partial^{2} r}{\partial z^{2}}\right\} \\
& +\frac{\Delta t^{4} V^{4}}{12}\left\{A^{2} \frac{\partial^{4} p}{\partial x^{4}}+A B \frac{\partial^{4} r}{\partial x^{2} \partial z^{2}}+B^{2} \frac{\partial^{4} p}{\partial x^{2} \partial z^{2}}+B \frac{\partial^{4} r}{\partial z^{4}}\right\} \\
R_{i, j}^{n+1}= & 2 R_{i, j}^{n}-R_{i, j}^{n-1}+\Delta t^{2} V^{2}\left\{B \frac{\partial^{2} p}{\partial x^{2}}+\frac{\partial^{2} r}{\partial z^{2}}\right\} \\
& +\frac{\Delta t^{4} V^{4}}{12}\left\{A B \frac{\partial^{4} p}{\partial x^{4}}+B^{2} \frac{\partial^{4} r}{\partial x^{2} \partial z^{2}}+B \frac{\partial^{4} p}{\partial x^{2} \partial z^{2}}+\frac{\partial^{4} r}{\partial z^{4}}\right\}
\end{aligned}\right.
$$

where $P=\left[\begin{array}{lll}P & \frac{\partial P}{\partial x} & \frac{\partial P}{\partial z}\end{array}\right]^{T}, R=\left[\begin{array}{lll}R & \frac{\partial R}{\partial x} & \frac{\partial R}{\partial z}\end{array}\right]^{T}, A=1+2 \varepsilon, B=\sqrt{1+2 \delta}$, the required expressions of high-order spatial partial derivatives can be obtained by using the truncated Taylor-series expansion of $P$ and $R$ at grid points $A_{i+l, j+m}(l \in\{-1,0,1\}) \quad(m \in\{-1,0,1\})$.According to ONAD method[7], we can solve equation (5).

\section{Numerical experiments}

The first numerical experiment in this section is one homogenous isotropic media with a computational domain $10 \mathrm{~km} \times 10 \mathrm{~km}$. The computational parameters are chosen as the spatial steps $\Delta \mathrm{x}=\Delta \mathrm{z}=0.05 \mathrm{~km}$ and the time step $\Delta \mathrm{t}=0.005 \mathrm{~s}$. We will simulate the $\mathrm{qP}$-wave propagation in this 
section. The $\mathrm{qP}$-wave velocity $v_{p}=4 \mathrm{~km} / \mathrm{s}$. The anisotropy parameters are $\varepsilon=0.2, \delta=0.1$, respectively. An explosive force located at the center of computational domain. The source term is a Ricker wavelet with main frequency $f_{0}=20 \mathrm{~Hz}$.

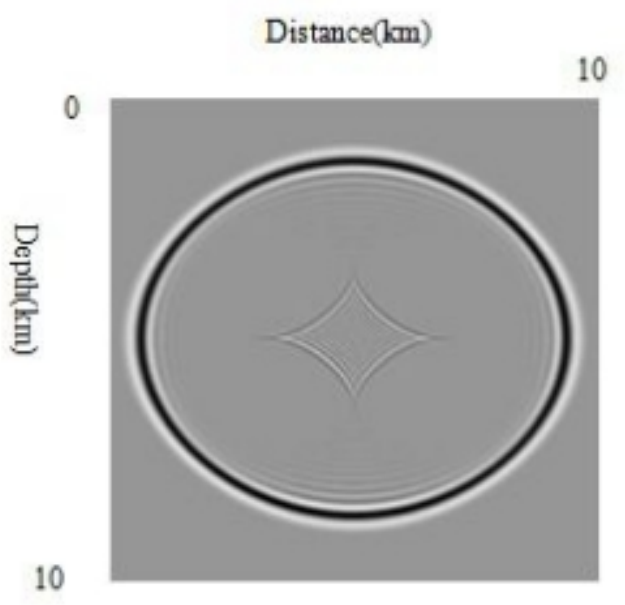

LWC

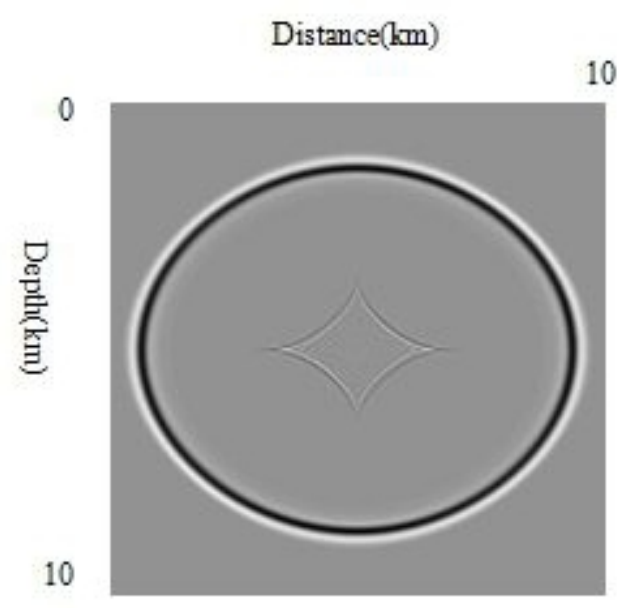

ONAD method

Fig.1 qP wave snapshots generated by LWC and ONAD method

Fig. 1 give the wavefield snapshots of qP-wave at 1s generated by LWC and ONAD method, respectively. From the wavefields, these two numerical results are almost same. However, the snapshot generated by ONADM is very clear without any visible numerical dispersion. The snapshot generated by LWC scheme has serious numerical dispersion. A diamond shape is observed at the center of the wavefield snapshot. It's the ghost of qSV wave which is not eliminated perfectly by acoustic approximation and it is nonphysical. It's harmful for the qP-wave's study.

There are two ways to eliminate the q-SV ghost effect, one is putting the source in a elliptical anisotropic layer and the other is putting the source in a isotropic subregion.

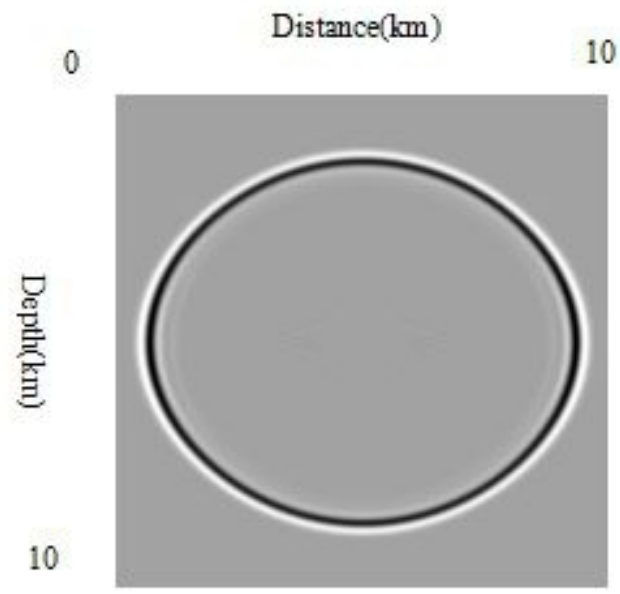

Elliptical anisotropy layer

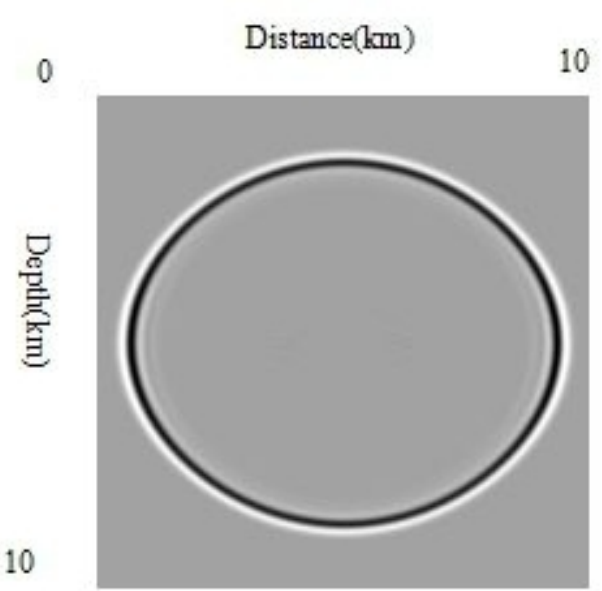

Isotropic layer

Fig.2 Suppress qSV wave

Fig. 2 is to simulate qP-wave propagation in homogeneous and isotropic media with a computational domain $10 \mathrm{~km} \times 10 \mathrm{~km}$. The computational parameters are chosen as the spatial steps $\Delta \mathrm{x}=\Delta \mathrm{z}=$ $0.025 \mathrm{~km}$ and the time step $\Delta \mathrm{t}=0.002 \mathrm{~s}$. The figure shows wave-field snapshots at time $1 \mathrm{~s}$. Fig. 2 shows isotropic layer and elliptic anisotropy layer can suppress qSV wave. 


\section{Conclusions}

An ONADM for 2D qP-wave propagation simulation in homogeneous media is proposed in this paper. ONADM can suppress the numerical dispersion successfully when coarse grids are used or strong velocity contrast is encountered. Compared with the traditional high order finite difference scheme LWC, ONADM's numerical results is satisfactory. We also use the isotropic sub domain technology to conquer the fake qSV-wave which is produced by extraneous root of the governing equations. Numerical results shows that this is a effective way to eliminate the fake qSV-wave.However, in order to remove the fake qSV-wave perfectly, the more research are need which will be our future study content.

\section{Acknowledgements}

This work was financially supported by the China Natural Science Foundation(41204074), the SWPU Youth Science and Technology Innovation team(2015CXTD07) of Seismic wave propagation and Imaging and SWPU Open Found (PLN146) of State Key Laboratory of Oil and Gas Reservoir Geology and Exploitation.

\section{References}

[1] Alkhalifah T. Acoustic approximations for processing in transversely isotropic media. Geophysics, 1998, 63(2): 623-631.

[2] Alkhalifah T. An acoustic wave equation for anisotropic media. Geophysics, 2000, 65(4): 1239-1250.

[3] Zhou H, Zhang G Q and Bloor R. An anisotropic acoustic wave equation for VTI media. 68th Annual International Conference and Exhibition, Extended Abstracts, H033. SEG, 2006.

[4] Duveneck E, Milcik P, Bakker P M, et al. Acoustic VTI wave equations and their application for anisotropic reverse-time migration,78th Ann. Internat. Mtg. Soc. Expl. Geophy., Expanded Abstracts, Las Vegas, 2008,27(1): 2186-2190.

[5] Du X, John C B and Larry R L. Anisotropic reverse time migration for tilted TI media.

Geophys. Prospect, 2007, 55(6): 853-869.

[6] Yang D, J Teng, Z Zhang and E Liu, A nearly analytic discrete method for acoustic and elastic wave equations in anisotropic media: Bull. Seis. Soc. Amer., 2003, 93, 882-890.

[7] Yang, D, G Song and M Lu, Optimally accurate nearly analytic discrete scheme for wave-field simulation in 3D anisotropic media: Bull. Seis. Soc. Amer., 2007, 97, 1557 - 1569.

[8] Tsvankin I. Seismic signatures and analysis of reflection data in anisotropic media. Elsevier.

[9] Thomsen L. Weak elastic anisotropy.Geophysics, 51(10): 1954-1966.

[10]Dablain M A. The application of high-order differencing to scalar wave equation. Geophysics, 1986, 51(1): 54-66. 